\title{
Scheduling design process with workflow for armored vehicle transmission
}

\author{
Zhibo Qiu ${ }^{1, a}$, Nan Nan ${ }^{2, ~ b}$, Yan Qin ${ }^{1, c}$, \\ ${ }^{1}$ China North Vehicle Research Institute, Beijing 100072, China \\ ${ }^{2}$ Jining Polytechnic, Jining 272073, China \\ azhbqiu@noveri.com.cn, bouttol@163.com, yanqin@noveri.com.cn
}

Keywords: Workflow, design process, optimizing, transmission, armored vehicle.

\begin{abstract}
Studying the scheduling problems of product design process is great significance to shorten the cycle of product development and to speed up product development. The problem of optimal scheduling of transmission design flow of armored vehicle can be regarded as an optimal problem of resource constrained project scheduling. Due to the complexity of the factors affecting project, the traditional perspective is difficult to establish the proper model. Through workflow modeling we can fully describe information of the processes. In this article, based on the problem of optimal scheduling of transmission design flow of armored vehicle, we studied scheduling optimization problems based on product design process of workflow, then provided the optimal model based on the product design process of workflow. At last, we developed a system platform based on product design project scheduling of workflow to allow users to define process of product design according to their requirements and to optimize the design task scheduling through optimal scheduling modules in the platform in order to achieve the goal of shortening project duration.
\end{abstract}

\section{Introduction}

Based on the problem of optimal scheduling of transmission design flow of armored vehicle, for these problem which can be regarded as an optimal problem of resource constrained project scheduling, we propose a scheduling model based on optimal process of product design of workflow, and design a scheduled platform of optimal process for the transmission design of armored vehicle.

The concept of workflow derives from production management, and WFMC (Workflow Management Coalition) define as that all or part of automation the business process; in the process, documents, information or tasks alternate in accordance with certain rules to achieve coordination among members of the organization in order to achieve business goals [1].

Product design process can be defined as a design task consisted of various activities connected each other of different functions, according to a certain logic and order in which they are executed. Figure 4.7 shows a lap joint design processes. Figure 1 shows that product design process consists of a series of design tasks, which are connected based on logical relationships.

\begin{tabular}{|c|c|c|c|c|}
\hline $\begin{array}{l}\text { Work out a } \\
\text { project plan }\end{array}$ & $\begin{array}{l}\text { Generate } \\
\text { concepts }\end{array}$ & $\begin{array}{c}\text { Estimate concept and choose } \\
\text { the best of them }\end{array}$ & $\begin{array}{c}\text { Estimate performance, manufacture, } \\
\text { assembly and costing }\end{array}$ & $\begin{array}{l}\text { Draw up } \\
\text { documents }\end{array}$ \\
\hline
\end{tabular}

Fig.1 Example of product design process

According to Figure 1, the workflow is an abstract overview of product design process, and workflow, as an indispensable part, is closely related to product design processes. In this paper, we focus on the scheduling optimization problems based on product design process of workflow.

Currently, for the research of model of workflow, the methods mainly include[2]: Process model based on activity network diagram[3]; Process chain model based on Event-driven[4]; Workflow model based on Petri net[5]; Workflow model based on speech act theory and Workflow transaction model, etc. But these workflow models generally have the following problems: (1) Representation are not intuitive; (2) the flexibility of model is not well, and uneasy to handle uncertain problems arising 
the implementation. (3) the model lacks of targeted: some models are too simple and unable to describe complex processes; in order to pursue commonality and universality, some models have powerful ability of description, though they are too complicated. For some problems, such as the problem of product design, those models lack of applicability; some others lack of steps for actual product design, such as Fallback, etc. (4) In order to meet the requirements of most fields for work flow, models commonly include the cycling route and polling the routing node, which does not apply to product design and resource-constrained scheduling optimization of project.

By analysis, these models cannot be used directly on product design process scheduling optimization problems, so in this paper, based on activity network diagram, we proposed scheduling optimization model for product design process of transmission of armored vehicle.

\section{Workflow model based on active network}

At present, there are many methods to build the workflow model: Workflow based on Petri net model; XPDL--Workflow modeling method based on XML; Based on the AON network method. Where, because AON network method have good logic and feasibility to cope with the problems proposed in this paper, so we choose AON network diagrams to build workflow models. Some concepts of the AON network diagram are defined as follows:

Definition 1: A directed graph $G$ represents the project implementation process, it is a tuple: $G=\langle N, C\rangle$

Where $\mathrm{N}$ is the set of all tasks in the project, known as the node; $\mathrm{C}$ is a collection of all arrows in the project, which represent the logical relationship between project tasks.

Definition 2: Node $\mathrm{N}$ is a task that is in the process of project implementation:

$N=\langle D, E, R, T\rangle$

Where, $\mathrm{D}$ are the input and output data sets; $\mathrm{E}$ is the performer of the task, and it can be people or a sector, and also can be a software component; $\mathrm{R}$ is a resources collection of task execution required; $\mathrm{T}$ is the logical constraints of task.

Definition 3: Time constraints:

$$
T=\left\langle t, t^{f s}, \bar{d}, f^{d}, t^{d i f}, t^{d i s}\right\rangle
$$

Where, $\mathrm{t}$ is the duration of task; $t^{f s}$ is the start time of force of the task, that is, task must be performed after the specified start time; $\bar{d}$ is the deadlines; $f^{d}$ is a fixed-time constraint, it represents the activity can only be implemented within the specified period of time; $t^{\text {dif }}$ is the time difference, which caused by two adjacent tasks in different time zones; $t^{\text {dis }}$ is the distance, which represents the required time interval between two tasks.

\section{Product design and process optimization scheduling model based on workflow}

Based on activity network diagram to build workflow models, and then constructing optimal scheduling model of product design process based on workflow, with its combined with XML, we define a kind of process execution Language for the design of transmission device, by which describes relationships and properties formally between objects, and provide scheduling model of product design process(as shown in the Figure 2)。

According to WfMC workflow reference model in the process of engine features description, one of them is the analysis process of defination. To satisfy this function, two prerequisites are necessary[6]:

(1) a formal description language (usually XML). Making use of this description language can describe a process definition.

(2) A set of objects can reflect the process definition model and results, generally called definition object. 


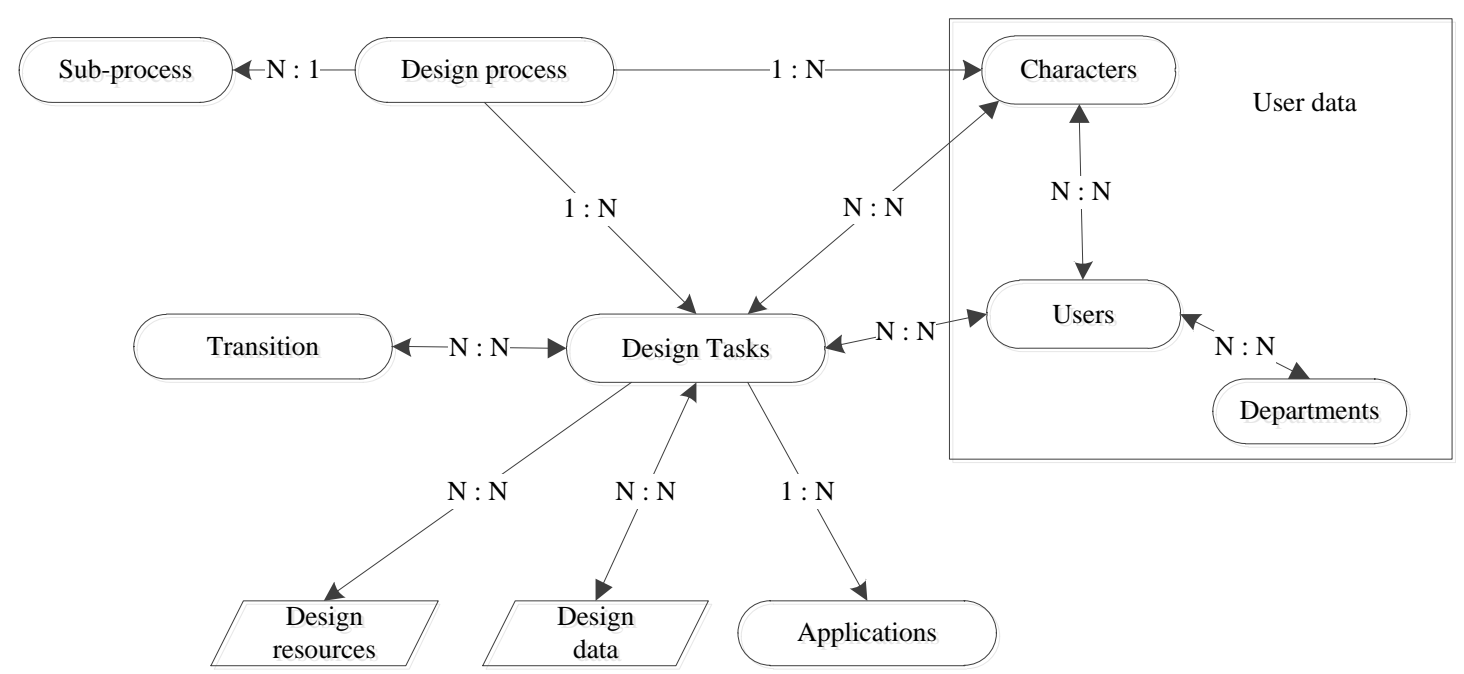

Fig.2 Meta-model of Product design Process Definition

\section{Achieve the optimal scheduling of Transmission design of armored vehicle based on workflow}

\subsection{Formal description of the design process}

As the element model shown in Figure 2, in the paper, we studied product design and scheduling model through the combination of elements, such as design process, child process, design task, jump, design resource, design data, application, roles, users and departments to describe the prodect design process. Making use of process scheduling engine parses the design process into a set of objects, and the group structure can reflect the design structure.

\subsection{The realization of graphical process design file generation tool}

When the process description is end, system should be called a graphical process design file generation tool to generate an XML format file, which are used to implement optimal scheduling of the product design 。 Based on the preceding analysis, when the process of product design is end and the results are saved to the database, the system will automatically generate the PDPEL grammar XML format and JPG image files, and save them to the database or local in order to optimize the scheduling and execution of this process.

\subsection{Realization of optimal scheduling modules}

When graphical process design document generation tool generates an XML format file, system calls optimization modules to analyze the XML file, and to optimize the design task scheduling in order to achieve the aim of shortening.Optimal operation interface of ZK as shown in Figure 3.

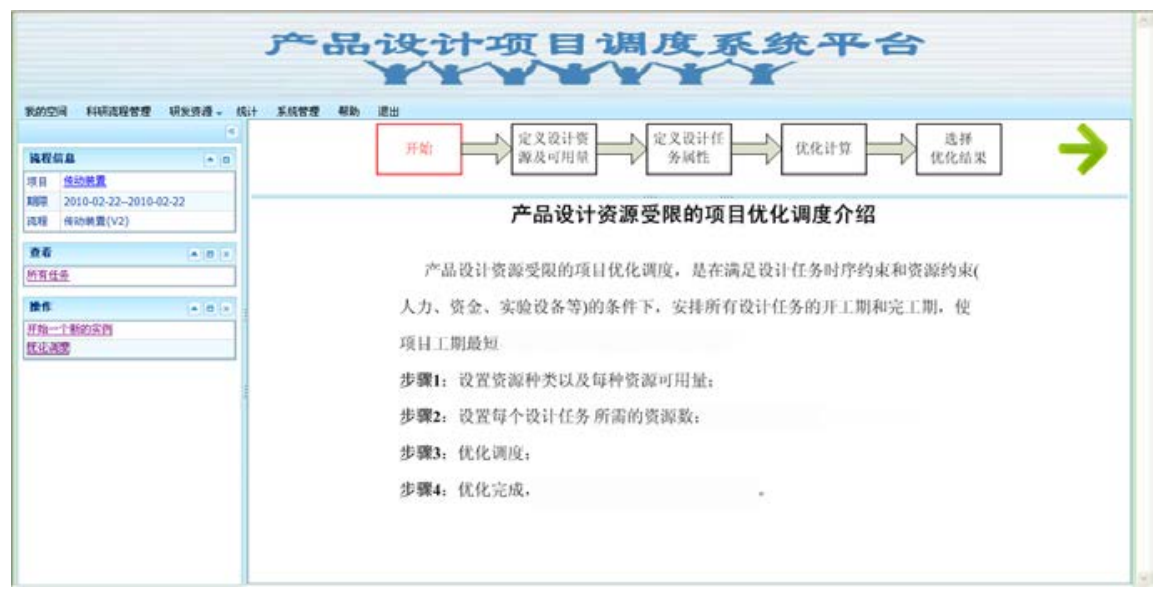

Fig.3 The interface of optimal design designed by ZK 


\section{Conclusion}

In order to solve the problem of armored vehicle transmission design process scheduling, this paper research the product design process based on the workflow scheduling optimization problem.

As discussed in the paper, the base of implementation of process scheduling is to build the correct model. The influencing factors of projects is very complicated, So it's very hard to establish the correct model from the traditional perspective. But through modeling of workflow can fully describe the information of process. Hence, it's is of great significance to research the product design process based on the workflow scheduling problem.

Also, the paper research and develop a product design project scheduling system platform based on workflow. Through this platform, users can define the product design process according to requirements. And then, through the scheduling optimization module of platform to optimize the design task scheduling in order to cut down project time.

\section{Acknowledgment}

This work is supposed by National Natural Science Foundation of China (Grant No.61304206).

\section{References}

[1]. Davis E. W., Patterson J. H. A Comparison of heuristic and optimum solutions in Resource-Constrained project scheduling [J]. Management Science, 1975, 21(8): 944-955.

[2]. Zhang Z H, Liu D Y. An Improved workflow manage system for ERP [J]. Application Research of Computers, 2008, 25(4): 1278-1280.

[3]. Browning T R, Ramasesh R V. A Survey of Activity Network - Based Process Models for Managing Product Development Projects[J]. Production and Operations Management, 2007, 16(2): 217-240.

[4]. Scheer A W, Thomas O, Adam O. Process modeling using event-driven process chains[J]. Process-Aware Information Systems, 2005: 119-146.

[5]. Chen J B, Han L, Xiong D Y, et al. Design and implementation of financial workflow model based on the petri net[M]//Advances in Future Computer and Control Systems. Springer Berlin Heidelberg, 2012: 495-500.

[6]. Hollings W D. The workflow reference model [M]. Workflow Management Coalition, 1995. 